\title{
Glia get rhythmic
}

Flies have been used to study the molecular basis of circadian behaviours since the early 1970s, and many genes that govern daily rhythms in Drosophila melanogaster, such as period (per), have been shown to be conserved in humans. The D. melanogaster brain contains populations of clock neurons that regulate dawn and dusk bouts of locomotor activity. In Current Biology, Jackson and colleagues now show that astrocytes have a key role in the regulation of this circadian behaviour.

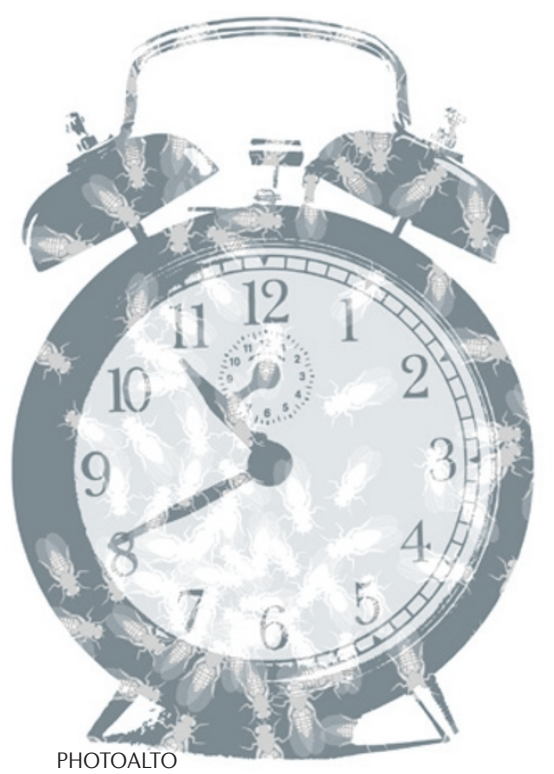

The authors expressed a bacterial sodium channel in adult glial cells to disrupt the cells' ionic gradient and, hence, their function. Expression of the sodium channels was temperature sensitive to avoid potential developmental effects. Following light-dark entrainment at $23^{\circ} \mathrm{C}$, both control and mutant flies displayed rhythmic locomotor activity. However, when these flies were exposed to a temperature of $30^{\circ} \mathrm{C}$, which allowed expression of the bacterial sodium channel, the mutant flies showed arrhythmic locomotor activity within 1 day. Disruption of vesicle trafficking (through expression of a temperature-sensitive dynamin molecule, Shibire ${ }^{\text {ts }}$ ) or an elevation of calcium levels in the glial cytoplasm (through knockdown of the sarco-endoplasmic reticulum calcium ATPase) caused a similar loss of rhythmicity in mutant flies. Together, these findings indicate that gliotransmission and calciumdependent pathways are important for the generation of this circadian behaviour.

To examine whether impairment of glial activity affected clock neurons, the authors quantified the abundance and cyclic expression of the clock proteins PER and PDP1ع, but they found no differences between the mutant and control flies at $30^{\circ} \mathrm{C}$. They did, however, find a marked decrease in the levels of the clock neuron peptide transmitter pigment dispersing factor (PDF) in projections of the ventral lateral pacemaker neurons in flies expressing Shibire ${ }^{\text {ts. }}$. These levels returned to normal when the temperature was decreased to $23^{\circ} \mathrm{C}$, suggesting that glia physiologically regulate neuronal PDF levels.

Five classes of glial cells exist in flies. Through the use of classspecific Gal4 drivers, Jackson and colleagues limited the expression of Shibire ${ }^{\text {ts }}$ to specific glial subpopulations. They showed that Shibire ${ }^{\text {ts }}$ expression in astrocytes was sufficient to disrupt circadian locomotor activity. Given that the regulation of circadian networks is highly conserved across species, it will be interesting to determine whether glia also have an important role in regulating circadian behaviour in mammals.

Monica Hoyos Flight

ORIGINAL RESEARCH PAPER Ng, F. S. et al.

Glial cells physiologically modulate clock neurons and circadian behavior in a calciumdependent manner. Curr. Biol. 14 Apr 2011 (doi:10.1016/j.cub.2011.03.02) 